\section{Engineering a dynamic model of the alveolar interface for the study of aerosol deposition}

\author{
Roberta Nossa, ${ }^{1,2}$ Joana Costa,1 \\ Ludovica Cacopardo,1 Arti Ahluwalia 1,2 \\ 1Research Center "E. Piaggio", \\ University of Pisa, Pisa; ${ }^{2}$ Department of \\ Information Engineering, University of \\ Pisa, Pisa, Italy
}

\begin{abstract}
Nano and micro particles are widely used in industrial, household and medicinal applications. To understand the interaction between particles and epithelial cells, we developed a dynamic model of the alveolar interface. This system, named DALI (Dynamic Model for the ALveolar Interface), is a modular bioreactor composed of two chambers divided by a porous membrane where epithelial lung cells are seeded. The membrane is the support of the alveolar barrier that separates the two compartments of the alveolus: the air and blood side. The system integrates the following elements: i) Air/Liquid interface, thanks to the two chambers divided by the membrane: ii) Cell culture media flow, thanks to the presence of a peristaltic pump; iii) Lung breathing motion, thanks to an airflow that allows the stretching of the membrane; iv) Aerosol deposition system, to study the effects of drug efficacy or particle toxicity on the epithelial layer; v) Quartz Crystal Microbalance, to quantify the amount of aerosolized particles.
\end{abstract}

\section{Introduction}

In order to improve the physiological relevance of the lung model and to investigate the deposition of aerosolised nanoparticles on the alveolar barrier, a bioreactor able to mimic breathing movements was designed. The system, named DALI (Dynamic model for ALveolar Interface), consists of a commercial aerosol generator, two bioreactors with a moving membrane placed between an air-liquid interface (Figure 1a), and a Quartz Crystal Microbalance (QCM) to measure the effective nanoparticle dose on the membrane (Figure 1c).

\section{Materials and Methods}

The bioreactor is composed of two polycarbonate cylindrical chambers (A and $B$ in Figure 1b): the upper one for the air flow (height: $24 \mathrm{~mm}$, diameter: $24 \mathrm{~mm}$ ) and the bottom one for the medium flow (height: $20 \mathrm{~mm}$, diameter: $24 \mathrm{~mm}$ ). Between them there is the porous stretchable membrane fixed in a holder that consists of two annular magnets covered by PDMS ( $\mathrm{C}$ in Figure 1b). The upper chamber is connected to an aerosol system for nanoparticles deposition (D in Figure $1 b$ ), and to a pressure regulator that ensures the cyclic stretching of the membrane. The electronics and the pressure regulators are placed in a control box. Potentiometers on the control box allow regulating the stretching level of the membrane for mimicking different stretching conditions.

The membrane was fabricated by electrospinning using a 1:1 (w/w) Bionate $\AA$ :Gelatin solution. The Bionate $\AA$ II $80 \mathrm{~A}$, a commercial poly(carbonate)urethane copolymer, was used to replicate the basement of the alveoli, as it guarantees membrane flexibility necessary to mimic the cyclic motion during the breathing. Additionally, in order to increase cell adhesion, gelatin was used in combination with Bionate ${ }^{\circledR}$ to obtain the final formulation for the membrane. Cell adhesion and biocompatibility were assessed by the Alamar Blue assay and cell staining with DAPI and rhodamine conjugated phalloidin.

A FEM model was used to simulate membrane displacement due to the pressure imbalance on the two sides of the air/liquid interface. The model was based on the Fluid Structure Interaction module of Comsol Multiphysics 4.3a software and consists of a cylindrical chamber connected to the external system with an inlet and an outlet tube ( $5 \mathrm{~mm}$ in diameter). The $80-\mu \mathrm{m}$ thick membrane was modelled as a disk on the top of the bioreactor and undergoes a constant pressure from the top. The inlet velocity was fixed at $100 \mu \mathrm{L} / \mathrm{min}$, and the fluid dynamics solved in the Laminar Flow regime. Bioreactor walls were set as walls with the no slip condition, and water chosen as a reference fluid. The FEM model was solved for different pressures ( 1 to $15 \mathrm{kPa}$ with a step of $1 \mathrm{kPa}$ ), in order to establish the pressure at which the membrane displacement in z-direction is $7 \mathrm{~mm}$, corresponding to a linear distention of $\approx 17 \%$, and so mimicking pathological levels of stretching. ${ }^{1,2}$
Correspondence: Roberta Nossa, Research Center "E. Piaggio", University of Pisa, Pisa, Italy.

E-mail: roberta.nossa@ing.unipi.it

Key words: In vitro model; alveolar interface; aerosol; dynamic model; bioreactor.

Conference presentation: this paper was presented at the Second Centro 3R Annual Meeting - 3Rs in Italian Universities, 2019, June 20-21, University of Genoa, Italy.

Received for publication: 28 October 2019. Accepted for publication: 11 November 2019.

This work is licensed under a Creative Commons Attribution NonCommercial 4.0 License (CC BY-NC 4.0).

C Copyright: the Author(s), 2019

Licensee PAGEPress, Italy

Biomedical Science and Engineering 2019; 3(s3):115 doi:10.4081/bse.2019.115

\section{Results}

In the DALI, the membrane is placed between annular magnets covered by PDMS. During static experiments, the holder with the membrane can be placed both in a 6-well multiwell, or inserted between the top and bottom chambers. The bioreactor is closed tightening wing nuts and the tightness of the bioreactor is ensured by the presence of the membrane holder enclosed in PDMS, which is self-adhesive and deformable. The bioreactor can be sterilized by ethanol solution, gas plasma, or ultraviolet light.

During dynamic experiments, the basolateral chamber is connected to a commercial peristaltic pump with an inlet tube, and to the reservoir with an outlet tube (Figure 1). The apical chamber is connected to the aerosol device and to a compressed air system, with an interposed pressure regulator put inside a control box. Potentiometers on the control box allow regulating the stretching level of the membrane: $<5 \%, 5-12 \%$, $12-17 \%$ and $>17 \%$ for mimicking different stretching conditions, both physiological and pathological.

A FEM model was used to simulate the velocity field of the fluid flowing through the bottom chamber, and to simulate the membrane displacement due to the pressure imbalance on the two sides of the air/liquid interface. When the pressure in the upper chamber increases, the membrane stretches, moving into the basolateral chamber, until it reaches an equilibrium with the hydrody- 
a)
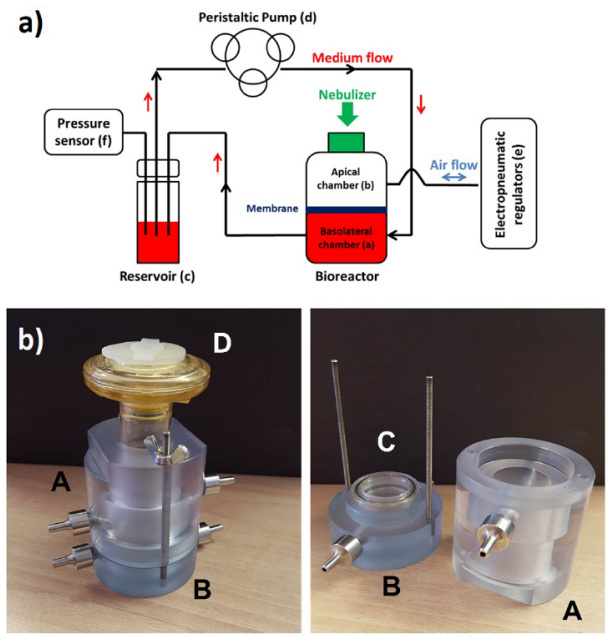

c)

A
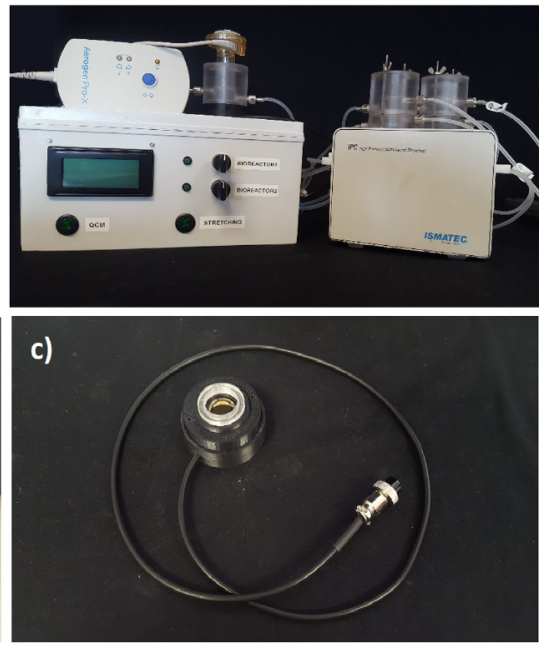

Figure 1. a) Schematic representation of the DALI system (on the left) and its picture (on the right); b) pictures of the component parts of the bioreactor; c) picture of the QCM device.

namic pressure of the flowing liquid. With the FEM model, it was possible to predict the pressure ranges that the external system must apply in order to achieve the desired stretching field on the membrane. The applied pressure corresponding to a z-displacement of almost $7 \mathrm{~mm}$ is $14 \mathrm{kPa}$. This displacement corresponds to a linear disten- tion of $\approx 17 \%$, and so mimicking pathological levels of stretching. 1,2

\section{Conclusions}

To conclude, we present a bioreactor that is able to replicate the cyclic motion during the breathing. The flexible moving membrane causes the rhythmic stimulation of epithelial cells, leading to the study of the interaction between them and the particles, in a system that replicates in vivo conditions. The electrospun 1:1 (w/w) Bionate ${ }^{\circledR}$ : gelatin membrane has been selected as suitable membrane for our application, as it is biocompatible and highly flexible, allowing physiological deformation levels. This study, based on the 3R's Statement, paves the way towards the development of an actuation device for physiologically relevant studies of aerosol and drug delivery and toxicology.

\section{References}

1. Patel HJ. Characterization and application of dynamic in vitro models of human airway. PhD thesis, Department of Biomedical Engineering, Utah State University, Logan, Utah.

2. Cavanaugh KJ, Margulies SS. Measurement of stretch-induced loss of alveolar epithelial barrier integrity with a novel in vitro method. Am J Physiol Cell Physiol 2002;283:C1801-8. 